\title{
UTILIZING PRINCIPAL SINGULAR VECTORS FOR TWO-DIMENSIONAL SINGLE FREQUENCY ESTIMATION
}

\author{
H. C. So, Frankie K.W. Chan, C. F. Chan and W. H. Lau \\ Department of Electronic Engineering, City University of Hong Kong, Kowloon, Hong Kong \\ email: hcso@ee.cityu.edu.hk, ckwf@hkexperts.com,itcfchan@cityu.edu.hk,itwhlau@cityu.edu.hk
}

\begin{abstract}
In this paper, frequency estimation of a twodimensional (2D) cisoid in the presence of additive white Gaussian noise is addressed. By utilizing the rank-one property of the $2 \mathrm{D}$ noise-free data matrix, the frequencies are estimated in a separable manner from the principal left and right singular vectors according to an iterative weighted least squares procedure. We have also derived the mean and variance expressions for the frequency estimates, which show that they are approximately unbiased and their accuracy achieves CramérRao lower bound (CRLB) at sufficiently high signalto-noise ratio conditions. Computer simulation results are included to corroborate the theoretical development as well as to contrast the performance of the proposed algorithm with the weighted phase averager and iterative quadratic maximum likelihood method as well as CRLB.
\end{abstract}

Index Terms - frequency estimation, twodimensional parameter estimation

\section{Introduction}

The problem of two-dimensional (2D) frequency estimation from a $2 \mathrm{D}$ data set has received considerable attention in the literature [1]-[3] because it has applications in many areas such as sonar, radar, wireless communications and biomedical engineering. In this paper, 2D frequency estimation of a complex sinusoid in additive noise is addressed. The observed $2 \mathrm{D}$ data set is

$$
\begin{aligned}
r_{m, n}= & s_{m, n}+q_{m, n}, \\
& m=1,2, \cdots, M, \quad n=1,2, \cdots, N
\end{aligned}
$$

where

$$
s_{m, n}=\gamma e^{j(\mu m+\nu n)}
$$

is the noise-free cisoid. The $\gamma$ is the unknown complex amplitude and $\mu \in(-\pi, \pi)$ and $\nu \in(-\pi, \pi)$ are the unknown frequencies, while $q_{m, n}$ is a zero-mean complex white Gaussian noise, that is, its real and imaginary parts are white processes with identical but unknown variances of $\sigma^{2} / 2$ and uncorrelated with each other.
The task is to find $\mu$ and $\nu$ from the $M N$ samples of $\left\{r_{m, n}\right\}$.

Maximum likelihood (ML) frequency estimates for a complex tone can be obtained from the $2 \mathrm{D}$ periodogram maximum [1]. However, the ML estimator requires extensive computations and thus is unsuitable for real-time applications. To avoid high computational cost, phase-based [4] and linear prediction (LP) [5] approaches are two possible choices. As an example of the former methodology, Kay and Nekovei [2] have extended the weighted phase averager (WPA) [6], which utilizes the differenced phase data for one-dimensional (1D) frequency estimation, to 2D. While for the latter, an iterative quadratic maximum likelihood (IQML) estimator [3] which makes use of the LP property and weighted least squares (WLS) is devised recently. The IQML method can also be considered as the 1D extension of the generalized weighted linear predictor (WLP) [7]. In this work, we exploit the principal singular vectors of the 2D data matrix constructed from $\left\{r_{m, n}\right\}$ to achieve efficient frequency estimation.

The rest of the paper is organized as follows. The algorithm development is presented in Section 2. Based on the rank-one property of the $2 \mathrm{D}$ noise-free data matrix, we propose to find $\mu$ and $\nu$ from the principal left and right singular vectors, respectively, in a separable manner, according to an iterative WLS procedure which is similar to [7]. Mean and variance analysis of the proposed estimator is provided in Section 3. Numerical examples are included in Section 4 to corroborate the theoretical development and to evaluate the performance of our developed scheme by comparing with the WPA [2] and IQML [3] method as well as Cramér-Rao lower bound (CRLB). Finally, conclusions are drawn in Section 5 .

\section{Algorithm Development}

We first express (1)-(2) in matrix form as:

$$
\mathbf{R}=\mathbf{S}+\mathbf{Q}
$$

where $\mathbf{R} \in \mathcal{C}^{M \times N}, \mathbf{S} \in \mathcal{C}^{M \times N}$ and $\mathbf{Q} \in \mathcal{C}^{M \times N}$. Denoting the $(m, n)$ entry of $\mathbf{R}$ by $[\mathbf{R}]_{m, n}$, we have $[\mathbf{R}]_{m, n}=r_{m, n},[\mathbf{S}]_{m, n}=s_{m, n}$ and $[\mathbf{Q}]_{m, n}=q_{m, n}$. 
From the regular structure of $\mathbf{S}$, it is clear that the noise-free data matrix can be represented as

$$
\mathbf{S}=\gamma \mathbf{g h}^{T}
$$

where

$$
\mathbf{g}=\left[\begin{array}{llll}
e^{j \mu} & e^{j 2 \mu} & \cdots & e^{j M \mu}
\end{array}\right]^{T}
$$

and

$$
\mathbf{h}=\left[\begin{array}{llll}
e^{j \nu} & e^{j 2 \nu} & \cdots & e^{j N \nu}
\end{array}\right]^{T}
$$

are complex vectors of unity-magnitude tones with frequencies $\mu$ and $\nu$, respectively. Thus $\mathbf{g}$ and $\mathbf{h}$ satisfy the LP property:

$$
[\mathbf{g}]_{m} \quad=e^{j \mu}[\mathbf{g}]_{m-1}, \quad m=2,3, \cdots, M
$$

and

$$
[\mathbf{h}]_{n}=e^{j \nu}[\mathbf{h}]_{n-1}, \quad n=2,3, \cdots, N
$$

where []$_{m}$ represents the $m$ th element in the vector.

On the other hand, $\mathbf{R}$ can be decomposed using singular value decomposition (SVD) as

$$
\mathbf{R}=\mathbf{U} \boldsymbol{\Lambda} \mathbf{V}^{H}
$$

where $\boldsymbol{\Lambda}=\operatorname{diag}\left(\lambda_{1}, \lambda_{2}, \ldots, \lambda_{N}\right)$ is the diagonal matrix of singular values of $\mathbf{R}$ with $\lambda_{1} \geq \lambda_{2} \geq \cdots=$ $\lambda_{N} \geq 0$ while $\mathbf{U}=\left[\begin{array}{llll}\mathbf{u}_{1} & \mathbf{u}_{2} & \cdots & \mathbf{u}_{N}\end{array}\right] \in \mathcal{C}^{M \times N}$ and $\tilde{\mathbf{V}}=\left[\begin{array}{llll}\mathbf{v}_{1} & \mathbf{v}_{2} & \cdots & \mathbf{v}_{N}\end{array}\right] \in \mathcal{C}^{N \times N}$ are orthonormal matrices whose columns are the corresponding left and right singular vectors, respectively. From the decomposition in (4)-(6), it is obvious that $\operatorname{rank}(\mathbf{S})=1$ and thus the best rank-one approximation of $\mathbf{S}$ according to (9), denoted by $\hat{\mathbf{S}}$, is

$$
\hat{\mathbf{S}}=\lambda_{1} \mathbf{u}_{1} \mathbf{v}_{1}^{H}
$$

Comparing (4) and (10), it is obvious that $\lambda_{1}, \mathbf{u}_{1}$ and $\mathbf{v}_{1}^{*}$, correspond to $\alpha, \mathbf{g}$ and $\mathbf{h}$, up to an unknown multiplying constant, respectively. This means that the noise-free versions of $\mathbf{u}_{1}$ and $\mathbf{v}_{1}$, denoted by $\overline{\mathbf{u}}_{1}$ and $\overline{\mathbf{v}}_{1}$, possess the same LP property as in (7)-(8). Define

$$
\begin{aligned}
& \mathbf{x}_{1}=\left[\begin{array}{llll}
{\left[\mathbf{u}_{1}\right]_{1}} & {\left[\mathbf{u}_{1}\right]_{2}} & \cdots & {\left[\mathbf{u}_{1}\right]_{M-1}}
\end{array}\right]^{T} \\
& \mathbf{x}_{2}=\left[\begin{array}{llll}
{\left[\mathbf{u}_{1}\right]_{2}} & {\left[\mathbf{u}_{1}\right]_{3}} & \cdots & {\left[\mathbf{u}_{1}\right]_{M}}
\end{array}\right]^{T}
\end{aligned}
$$

and let $a=e^{j \mu}$, we then have:

$$
\mathbf{x}_{1} a \approx \mathbf{x}_{2}
$$

Following [7], the WLS estimate of $a$ is computed as:

$$
\begin{aligned}
\hat{a} & =\arg \min _{\tilde{a}}\left(\mathbf{x}_{1} \tilde{a}-\mathbf{x}_{2}\right)^{H} \mathbf{W}_{M}(a)\left(\mathbf{x}_{1} \tilde{a}-\mathbf{x}_{2}\right) \\
& =\frac{\mathbf{x}_{1}^{H} \mathbf{W}_{M}(a) \mathbf{x}_{2}}{\mathbf{x}_{1}^{H} \mathbf{W}_{M}(a) \mathbf{x}_{1}}
\end{aligned}
$$

The optimum weighting matrix, $\mathbf{W}_{M}(a) \in \mathcal{C}^{(M-1) \times(M-1)}$, is constructed from the residual error of $\mathbf{x}_{1} a-\mathbf{x}_{2}=\Delta \mathbf{x}_{1} a-\Delta \mathbf{x}_{2}$ where $\boldsymbol{\Delta}$ denotes the perturbation, and hence a function of $a$, which is commonly known as the Markov estimate [8].

With the use of (11)-(12), the inverse of $\mathbf{W}_{M}(a)$ is

$$
\begin{aligned}
\mathbf{W}_{M}^{-1}(a) & =E\left\{\left(\Delta \mathbf{x}_{1} a-\Delta \mathbf{x}_{2}\right)\left(\Delta \mathbf{x}_{1} a-\Delta \mathbf{x}_{2}\right)^{H}\right\} \\
& =\mathbf{A} E\left\{\boldsymbol{\Delta} \mathbf{u}_{1} \Delta \mathbf{u}_{1}^{H}\right\} \mathbf{A}^{H}
\end{aligned}
$$

where

$$
\mathbf{A}=\left[\begin{array}{ccccc}
1 & a & 0 & \cdots & 0 \\
0 & 1 & a & \cdots & 0 \\
\ddots & \ddots & \ddots & \ddots & \\
0 & 0 & \cdots & 1 & a
\end{array}\right] \in \mathcal{C}^{(M-1) \times M}
$$

and $E$ denotes the expectation operator. Employing [9], $\boldsymbol{\Delta} \mathbf{u}_{1}$ is approximated as:

$$
\begin{aligned}
\boldsymbol{\Delta} \mathbf{u}_{1} & \approx \bar{\lambda}_{1}^{-1} \overline{\mathbf{U}}_{n} \overline{\mathbf{U}}_{n}^{H} \mathbf{Q} \overline{\mathbf{v}}_{1} \\
& =\bar{\lambda}_{1}^{-1}\left(\overline{\mathbf{v}}_{1}^{T} \otimes \overline{\mathbf{U}}_{n} \overline{\mathbf{U}}_{n}^{H}\right) \operatorname{vec}(\mathbf{Q})
\end{aligned}
$$

where $\overline{\mathbf{U}}_{n}=\left[\begin{array}{llll}\overline{\mathbf{u}}_{2} & \overline{\mathbf{u}}_{3} & \cdots & \overline{\mathbf{u}}_{N}\end{array}\right]$ corresponds to the noisefree noise subspace and $\otimes$ and vec represent Kronecker product and vectorization, respectively. Employing (17), $E\left\{\boldsymbol{\Delta} \mathbf{u}_{1} \boldsymbol{\Delta} \mathbf{u}_{1}^{H}\right\}$ is derived as:

$$
\begin{aligned}
& E\left\{\boldsymbol{\Delta} \mathbf{u}_{1} \boldsymbol{\Delta} \mathbf{u}_{1}^{H}\right\} \\
= & \bar{\lambda}_{1}^{-2}\left(\overline{\mathbf{v}}_{1}^{T} \otimes \overline{\mathbf{U}}_{n} \overline{\mathbf{U}}_{n}^{H}\right) E\left\{\operatorname{vec}(\mathbf{Q}) \operatorname{vec}(\mathbf{Q})^{H}\right\} \times \\
& \quad\left(\overline{\mathbf{v}}_{1}^{*} \otimes \overline{\mathbf{U}}_{n} \overline{\mathbf{U}}_{n}^{H}\right) \\
= & \bar{\lambda}_{1}^{-2}\left(\overline{\mathbf{v}}_{1}^{T} \otimes \overline{\mathbf{U}}_{n} \overline{\mathbf{U}}_{n}^{H}\right)\left(\sigma^{2} \mathbf{I}\right)\left(\overline{\mathbf{v}}_{1}^{*} \otimes \overline{\mathbf{U}}_{n} \overline{\mathbf{U}}_{n}^{H}\right) \\
= & \bar{\lambda}_{1}^{-2} \sigma^{2}\left(\overline{\mathbf{v}}_{1}^{T} \overline{\mathbf{v}}_{1}^{*}\right) \otimes\left(\overline{\mathbf{U}}_{n} \overline{\mathbf{U}}_{n}^{H} \overline{\mathbf{U}}_{n} \overline{\mathbf{U}}_{n}^{H}\right) \\
= & \bar{\lambda}_{1}^{-2} \sigma^{2} \overline{\mathbf{U}}_{n} \overline{\mathbf{U}}_{n}^{H}
\end{aligned}
$$

where $\mathbf{I}$ is the identity matrix. Note that the values of $\bar{\lambda}_{1}$ and $\sigma^{2}$ are not required as they will be canceled out in (14), that is, we only need to know $\mathbf{W}_{M}(a)$ up to a multiplying scalar. Substituting (18) into (15) and with the use of $\mathbf{A} \overline{\mathbf{u}}_{1}=\mathbf{0}$ where $\mathbf{0}$ is the zero vector, and $\overline{\mathbf{U}}_{n} \overline{\mathbf{U}}_{n}^{H}=\mathbf{I}-\overline{\mathbf{u}}_{1} \overline{\mathbf{u}}_{1}^{H}, \mathbf{W}_{M}(a)$ is simplified as

$$
\begin{aligned}
\mathbf{W}_{M}(a) & =\left(\mathbf{A} \overline{\mathbf{U}}_{n} \overline{\mathbf{U}}_{n}^{H} \mathbf{A}^{H}\right)^{-1} \\
& =\left(\mathbf{A} \mathbf{A}^{H}\right)^{-1}
\end{aligned}
$$

Changing the variable from $a$ back to $\mu$ with $a=e^{j \mu}$ yields a closed-form expression for $\mathbf{W}_{M}(a)=\mathbf{W}_{M}(\mu)$ with elements [7]:

$$
\left[\mathbf{W}_{M}(\mu)\right]_{m, n}=\frac{M \min (m, n)-m n}{M} e^{j(n-m) \mu}
$$


where $\min (m, n)=m$ if $m<n$ and it is equal to $n$ otherwise. As $\mathbf{x}_{1}^{H} \mathbf{W}_{M}(\mu) \mathbf{x}_{1}$ is real and positive [7], we simplify (14) to obtain the WLS estimate of $\mu$, denoted by $\hat{\mu}$, as:

$$
\hat{\mu}=\angle\left(\mathbf{x}_{1}^{H} \mathbf{W}_{M}(\mu) \mathbf{x}_{2}\right)
$$

As $\mathbf{W}_{M}(\mu)$ is characterized by the unknown parameter $\mu$, we follow [7] to estimate $\mu$ in an iterative manner as follows.

(i) Obtain an initial frequency estimate using (21) with $\left[\mathbf{W}_{M}(\mu)\right]_{m, n}=0$ for $m \neq n$, which is in fact the WLP estimate [6].

(ii) Use $\hat{\mu}=\mu$ to construct $\mathbf{W}_{M}(\mu)$ according to (20).

(iii) Compute an updated $\hat{\mu}$ using (21).

(iv) Repeat Steps (ii)-(iii) until a stopping criterion is reached.

In a similar manner, $\nu$ is estimated independently from $\mathbf{v}_{1}$ according to

$$
\hat{\nu}=-\angle\left(\mathbf{y}_{1}^{H} \mathbf{W}_{N}(\nu) \mathbf{y}_{2}\right)
$$

where

$$
\mathbf{y}_{1}=\left[\begin{array}{llll}
{\left[\mathbf{v}_{1}\right]_{1}} & {\left[\mathbf{v}_{1}\right]_{2}} & \cdots & {\left[\mathbf{v}_{1}\right]_{N-1}}
\end{array}\right]^{T}
$$

and

$$
\mathbf{y}_{2}=\left[\begin{array}{llll}
{\left[\mathbf{v}_{1}\right]_{2}} & {\left[\mathbf{v}_{1}\right]_{3}} & \cdots & {\left[\mathbf{v}_{1}\right]_{N}}
\end{array}\right]^{T}
$$

\section{Performance Analysis}

The means and variances of $\hat{\mu}$ and $\hat{\nu}$ upon parameter convergence are now analyzed. To simplify the derivation, it is assumed that the signal-to-noise ratio (SNR) is sufficiently high such that $\hat{\mu} \rightarrow \mu$ and $\hat{\nu} \rightarrow \nu$ and we substitute the true frequencies for the estimates in the weighting matrices of $\mathbf{W}_{M}(\mu)$ and $\mathbf{W}_{N}(\nu)$.

First we consider $\hat{\mu}$. Our strategy is to decompose $\hat{\mu}$ as $\hat{\mu}=\mu+\Delta \mu$ where $\Delta \mu=\angle\left(e^{-j \mu} \mathbf{x}_{1}^{H} \mathbf{W}_{M}(\mu) \mathbf{x}_{2}\right)$ is the estimation error in $\hat{\mu}$, and then compute $E\{\Delta \mu\}$ and $E\left\{(\Delta \mu)^{2}\right\}$. Following the results in [7], it is shown that $E\{\Delta \mu\} \approx 0$ which implies that $E\{\hat{\mu}\} \approx \mu$, while its variance or mean square error (MSE), denoted by $\operatorname{var}(\hat{\mu})$, is

$$
\operatorname{var}(\hat{\mu})=\frac{\sigma^{2}}{2 \bar{\lambda}_{1}^{2} \overline{\mathbf{x}}_{1}^{H} \mathbf{W}_{M}(\mu) \overline{\mathbf{x}}_{1}}
$$

Furthermore, $\overline{\mathbf{x}}_{1}$ can be expressed as $\overline{\mathbf{x}}_{1}=$ $\mathbf{g}_{1} \exp \left(j \varphi_{g}\right) /|\mathbf{g}|$ where $\varphi_{g}$ is an unknown phase and $\mathbf{g}_{1}=\left[\begin{array}{llll}e^{j \mu} & e^{j 2 \mu} & \cdots & e^{j(M-1) \mu}\end{array}\right]^{T}$. With the use of $|\mathbf{g}|=\sqrt{M},|\mathbf{h}|=\sqrt{N}, \bar{\lambda}_{1}=|\mathbf{g}||\mathbf{h}||\gamma|=\sqrt{M N}|\gamma|$ and
$\mathbf{g}_{1}^{H} \mathbf{W}_{M}(\mu) \mathbf{g}_{1}=M\left(M^{2}-1\right) / 12[7],(25)$ can be simplified as:

$$
\operatorname{var}(\hat{\mu}) \approx \frac{6}{\operatorname{SNR} N M\left(M^{2}-1\right)}
$$

with SNR $=\left|\gamma^{2}\right| / \sigma^{2}$, which is the CRLB for $\mu$. Likewise, the estimate of $\nu$ is also approximately unbiased and its variance, denoted by $\operatorname{var}(\hat{\nu})$, is:

$$
\operatorname{var}(\hat{\nu}) \approx \frac{6}{\operatorname{SNR} M N\left(N^{2}-1\right)}
$$

which is the CRLB for $\nu$. That is, the 2D frequency estimator can attain ML performance when the SNR is high enough.

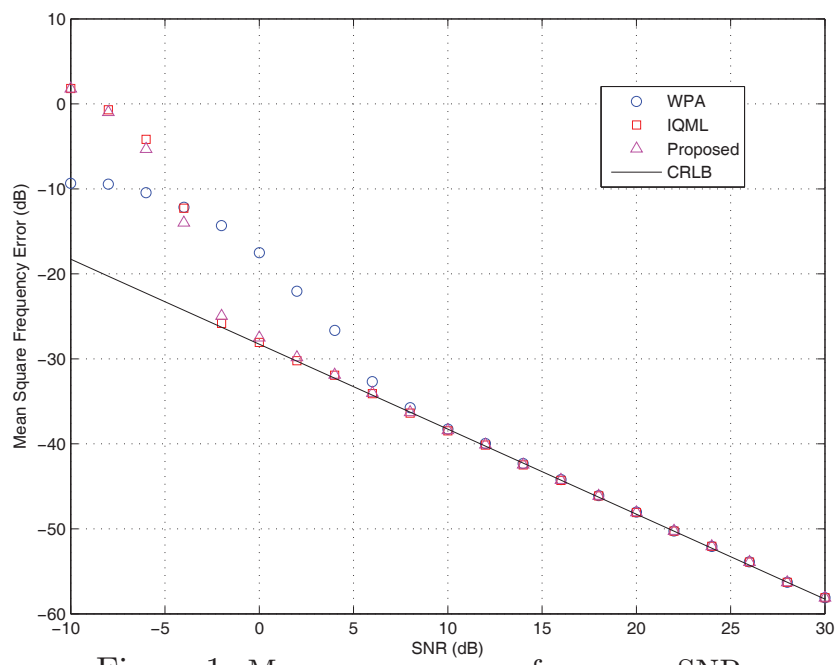

Figure 1: Mean square error of $\mu$ versus SNR

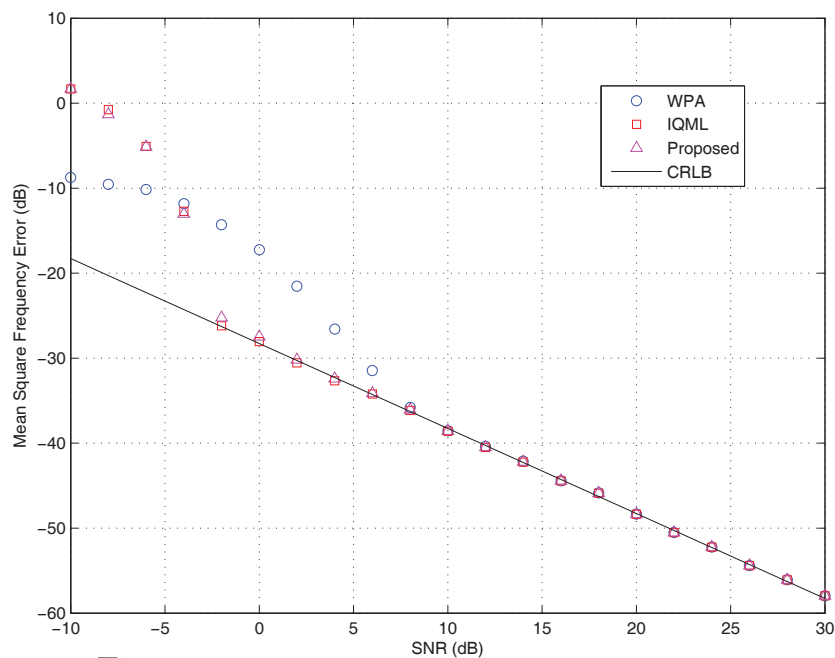

Figure 2: Mean square error of $\nu$ versus SNR

\section{Numerical Examples}

Computer simulations have been carried out to assess the estimation performance of the proposed SVD-based 


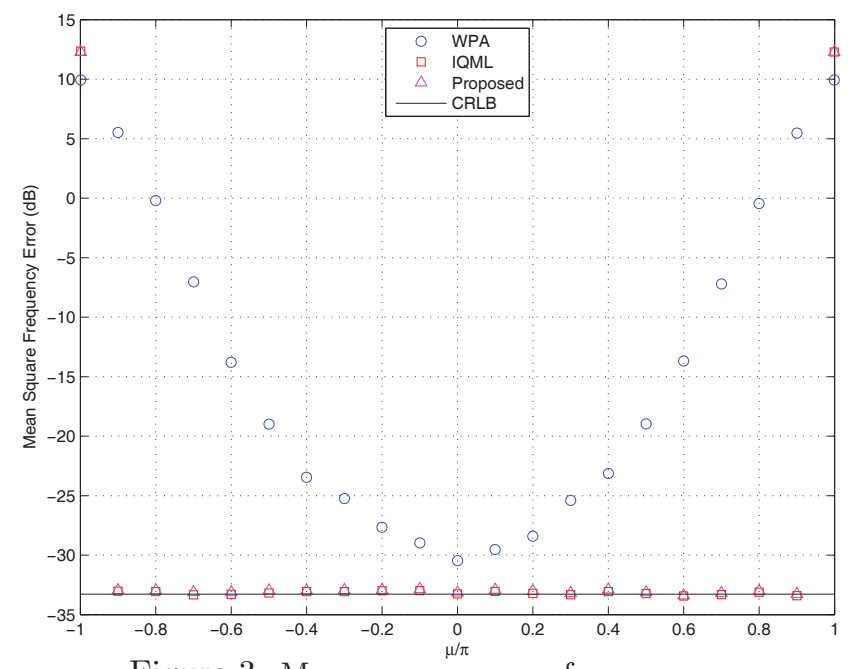

Figure 3: Mean square error of $\mu$ versus $\mu$

algorithm for a single 2D cisoid in white Gaussian noise. We compare its MSE performance with that of the WPA [2] and IQML [3] method as well as CRLB. We choose the stopping criterion of 3 iterations in both proposed and IQML schemes as no obvious improvement is observed for more iterations. The tone amplitude is $\gamma=1$ and $M=N=8$ while different SNRs are obtained by proper scaling the noise variance $\sigma^{2}$. All results are based on averages of 500 independent runs.

Figures 1 and 2 show the MSEs of $\mu$ and $\nu$ at $\mu=\nu=0.1 \pi$, respectively, for the WPA, IQML and proposed estimators as well CRLB versus SNR. Although all methods provide optimum accuracy at sufficiently high SNRs, the IQML and proposed estimators perform similarly and are superior to the WPA in terms of threshold SNR. That is, the former have a threshold SNR of $-2 \mathrm{~dB}$ while that of the latter is $8 \mathrm{~dB}$. The MSE performance of $\mu$ versus $\mu \in(-\pi, \pi)$ with $\nu=0.1 \pi$ is investigated in Figure 3. We see that both IQML and proposed estimators can achieve CRLB when $\mu$ is not approaching $-\pi$ or $\pi$, indicating their uniform estimation performance. While the WPA is suboptimal and cannot give uniform accuracy, which agrees with the study in [10]. From Figures 1 to 3, we also see that (26) and (27) are validated for sufficiently high SNR conditions.

Regarding computational load, the complexities of WPA and IQML method are $\mathcal{O}(M N)$ and $\mathcal{O}\left(M^{2} N^{2}\right)$, respectively, while the proposed method requires $\mathcal{O}\left(M^{3}\right)$ operations, assuming that $M \geq N$, due to the SVD and WLS procedures. As a result, the computational requirement of our scheme is in between those of WPA and IQML estimator when $M$ and $N$ are com- parable. The average computation times of the WPA, IQML and proposed estimators for a single trial are measured as $5.65 \times 10^{-5} \mathrm{~s}, 1.15 \times 10^{-3} \mathrm{~s}$ and $5.73 \times 10^{-4} \mathrm{~s}$, respectively, which agree with our complexity analysis.

\section{Conclusion}

An efficient frequency estimation approach for a twodimensional (2D) single complex tone in additive white Gaussian noise has been devised. The key ideas are to make use of the rank-one property of the 2D noise-free data matrix and find the two frequency parameters from the principal left and right singular vectors in a separable manner according to an iterative weighted least squares procedure. Mean and variance expressions for the frequency parameters are also produced and verified via computer simulations, which illustrate that they are approximately unbiased and their variance achieves Cramér-Rao lower bound at sufficiently large signal-tonoise ratio (SNR). Regarding comparative performance, the proposed scheme is superior to [2] in terms of threshold SNR at the expense of higher complexity. On the other hand, it has the same threshold behavior with [3] but the former is computationally less demanding.

\section{References}

[1] S.M.Kay, Modern Spectral Estimation : Theory and Application, Englewood Cliffs, NJ: Prentice-Hall, 1988

[2] S.Kay and R.Nekovei, "An efficient two-dimensional frequency estimator," IEEE Trans. Acoust., Speech, Signal Processing, vol.38, pp.1807-1809, Oct. 1990

[3] H.C.So and K.W.Chan, "Approximate maximum likelihood algorithms for two-dimensional frequency estimation of a complex sinusoid," IEEE Trans. Signal Processing, vol.54, no.8, pp.3231-3237, Aug. 2006

[4] S.A.Tretter, "Estimating the frequency of a noisy sinusoid by linear regression," IEEE Trans. Inform. Theory, vol.31, pp.832-835, Nov. 1985

[5] G.W.Lank, I.S.Reed and G.E.Pollon, "A semicoherent detection and Doppler estimation statistic," IEEE Trans. Aerosp. Electron. Syst., vol.9, pp.151-165, Mar. 1973

[6] S.Kay, "A fast and accurate single frequency estimator," IEEE Trans. Acoust., Speech, Signal Processing, vol.37, pp.1987-1990, Dec. 1989

[7] H.C.So and K.W.Chan, "A generalized weighted linear predictor frequency estimation approach for a complex sinusoid," IEEE Trans. Signal Processing, vol.54, no.4, pp.13041315, Apr. 2006

[8] T.Soderstrom and P.Stoica, System Identification, Englewood Cliffs, NJ: Prentice-Hall, 1989

[9] J.Liu, X.Liu and X.Ma, "First-order perturbation analysis of singular vectors in singular value decomposition," IEEE Trans. Signal Processing, vol.56, no.7, pp.3044-3049, Jul. 2008

[10] B.G.Quinn and E.J.Hannan, The Estimation and Tracking of Frequency, NY: Cambridge University Press, 2001 